\title{
First International Workshop on Model Based Engineering for Robotics (RoSym'10)
}

\author{
Laurent Rioux ${ }^{1}$, Davide Brugali ${ }^{2}$, and Sébastien Gérard ${ }^{3}$ \\ ${ }^{1}$ Decision Technologies and Mathematics Lab., Thales Research \& Technology, Campus \\ Polytechnique, 1, avenue Augustin Fresnel 91767 Palaiseau cedex France \\ laurent.rioux@thalesgroup.com \\ ${ }^{2}$ Dept. Computer Science and Mathematics, Università degli Studi di Bergamo, \\ v.le Marconi, 5, 24044 Dalmine, Italy \\ brugali@unibg.it \\ ${ }^{3}$ CEA LIST, Laboratoire d'Ingénierie dirigée par les modèles pour les Systèmes Embarqués \\ (LISE), Point Courrier 94, Gif-sur-Yvette, F-91191 France \\ sebastien.gerard@cea.fr
}

The main objectives of this workshop are to organize common discussions within Model-base Engineering (MBE) and Robotics experts on how MBE can help robotics people and to share issues that robotics people have encountered with MBE. Current engineering approaches for robotic systems have indeed been demonstrated to be insufficient to bypass following constraints that robotics embedded systems are currently facing:

- the problem space is huge: as uncertainty of the environment and the number and type of resources available to the robot increase, the definition of the best matching between current situation and correct robot resource exploitation becomes overwhelming even for the most skilled robot engineer,

- the solution space is huge: in order to enhance robustness of complex robotic systems, existing cognitive methods and techniques need to exploit robotic-specific resources adequately. This means that the robotic system engineer should master highly heterogeneous technologies in order to integrate them in a consistent and effective way.

One ideal process for developing robotic software components is to enable the design and implementation of highly complex and robust robotic systems to involve in less effort as possible. Robotics systems are complex and embedded ones; thanks to MBE that has already demonstrated its efficiency on complex and embedded systems. We expect MBE to be a real promising solution for the development process of robotics software and systems.

Potentially, new MBE techniques have to be developed for robotics which can also be applicable to other domains. Since robotics is a very challenging domain, we are confident that new techniques may possibly open new way for Model Based Engineering. 